\title{
Effect of Mental Fatigue on Error Monitoring
}

\author{
Feng $\mathrm{MA}^{1,2}$, Yi XIAO${ }^{2}$, Gui $\mathrm{CAl}^{2}$, Feng-gang $\mathrm{XU}^{2,3}$ and Shan-guang \\ $\mathrm{CHEN}^{1,2, a, "}$ \\ ${ }^{1}$ School of Aerospace Engineering, Tsinghua University, Beijing, China \\ ${ }^{2}$ National Key Laboratory of Human Factors Engineering, China Astronaut Research \\ and Training Center, Beijing, China \\ ${ }^{3}$ School of Biology and Medical Engineering, Beihang University, Beijing, China \\ amfhorse@126.com \\ ${ }^{*}$ Corresponding author
}

Keywords: Error monitoring, Mental fatigue, Event-related potentials (ERPs), Error-related negativity (ERN).

\begin{abstract}
Performance monitoring tasks generate response-locked event-related potentials (ERPs), generated from anterior cingulate cortex (ACC) region of the medial prefrontal cortex. However, the quantitative relationship between mental fatigue and error monitoring has not been closely investigated, which is the purpose of this study. Twenty-one participants were asked to perform a 100-minute 2-back work memory task for inducing mental fatigue and assessing error monitoring. The results showed that the participants were less accurate and slower during mental fatigue. The error-related negativity (ERN) amplitudes were statistically reduced after carrying out the 2-back task. The current pattern of results provides evidence that sustained attention plays an important role in maintaining error monitoring.
\end{abstract}

\section{Introduction}

Human error is considered one of the primary contributors to accidents in complex work domains such as trucking, nuclear power plants, aviation and aerospace [1-3]. Many factors, including task characteristics, physical environment, and human system interaction, result in human error. An especially important factor is performance monitoring, which is vital for adapting one's own behavior and enabling effective cognitive control. Error monitoring and feedback processing are two important facets of performance monitoring $[4,5]$. As a critical function of the human cognitive system, error monitoring is the ability to monitor and evaluate the outcomes and consequences of behavior in order to optimize and adapt subsequent behavior to realize goals such as driving vehicles, the rendezvous and docking (RAD) in human spaceflight, and so on.

Error processing and monitoring usually accompanies a negative deflection appearing in the event-related potentials (ERPs) after the time an error is committed, called the error-related negativity (ERN) [6-9]. The ERN is a negative potential that has a peak amplitude as high as about $10 \mu \mathrm{V}$, and a peak latency of approximately 50-150 $\mathrm{ms}$ after the onset of electromyography activity associated with an erroneous response, which is located at the fronto-central scalp [10-12]. The ERN reflects an error monitoring system in the brain, including error detection and error evaluation. Error monitoring is known to involve the anterior cingulate cortex (ACC) region of the medial prefrontal cortex (PFC) [13]. 
A growing number of studies have investigated the cognitive mechanism of error monitoring by recording the ERN. They focused on the aspects of inhibitory control, conflict monitoring, and feedback processing [14-17]. Previous researchers examined the qualitative relationship between mental fatigue and error monitoring, suggesting that changes in task performance due to mental fatigue were accompanied by a decrease in ERN amplitude [14, 16-20]. However, the quantitative relationship between mental fatigue and error monitoring has been seldom examined $[15,21]$. There is a need to understand the neurophysiological mechanisms of the link between mental fatigue and error monitoring.

Traditionally, error monitoring has generally been examined during Stop-Signal, Go/Nogo, Flanker, oddball, and learning tasks [4, 10, 22-26]. We examined behavior and brain responses during a 100-minute 2-back work memory task for error monitoring and mental fatigue. The present study aims to extend the previous findings by evaluating differences between the first 10 minutes and the last 10 minutes on the 2-back task in error monitoring using ERN. The second aim of this study is to address the quantitative correlation of mental fatigue and error monitoring. Based on prior studies, we hypothesized that the fatigued group would show smaller amplitudes on ERP components in performing the task relative to the control group, and that behavioral response would be poorer in the fatigued group.

\section{Materials and Methods}

\section{Participants}

Twenty-one right-handed, neurologically normal male volunteers participated in the study. All participants were undergraduate or graduate students from the China Astronaut Research \& Training Center and Beihang University. They reported normal or corrected-to-normal vision and were aged between 19 and 34 years (mean age $26.2 \pm 3.6$ years). All of them provided written consent and were financially compensated for participation. The use of human participants followed the guidelines of the Helsinki Declaration and was approved by the Research Ethics Committee of China Astronaut Research and Training Center. Only participants with a minimum of 5 error trials which yielded distinct ERN peaks were included in further data analysis [27]. Four participants had to be excluded from further analysis due to data acquisition artifacts $(n=2)$, or due to committing less than 5 errors $(n=2)$. Finally, there were 17 participants in data analyzing.

\section{Task}

Fatigue is classified as either physical or mental, and mental fatigue is the focus of this research. In a mental-fatigue-inducing task session, participants performed 100 minutes of 2-back test trials [28], which could not be performed without using working memory. The 2-back test procedure is shown in Figure 1.

In the 2-back test, the stimuli were randomly presented Arabic numerals between 0 and 9 displayed in white against a black background on a 24-inch computer liquid crystal display (LCD) monitor (the refresh rate was $60 \mathrm{~Hz}$ ). At a viewing distance of 80 $\mathrm{cm}$, the size of each stimulus was $1.8 \mathrm{~cm}$ tall and $1.4 \mathrm{~cm}$ wide, and the visual angle was 1.3. Each Arabic numeral was presented for $500 \mathrm{~ms}$, and the inter-stimulus intervals (ISI) were $2,500 \mathrm{~ms}$. Participants were required to determine whether the current numeral and the penultimate one were equal, and asked to press the $\mathrm{F}$ key if equal and 
press the $\mathrm{J}$ key if unequal. The equal numbers were named matching stimuli, and the unequal ones were named mismatching stimuli. Matching and mismatching stimuli were randomly intermixed in presentation with a ratio of approximately $2: 1$. Stimuli presentation and behavioral data acquisition were implemented using E-Prime 2.0 (Psychology Software Tools, Inc.).

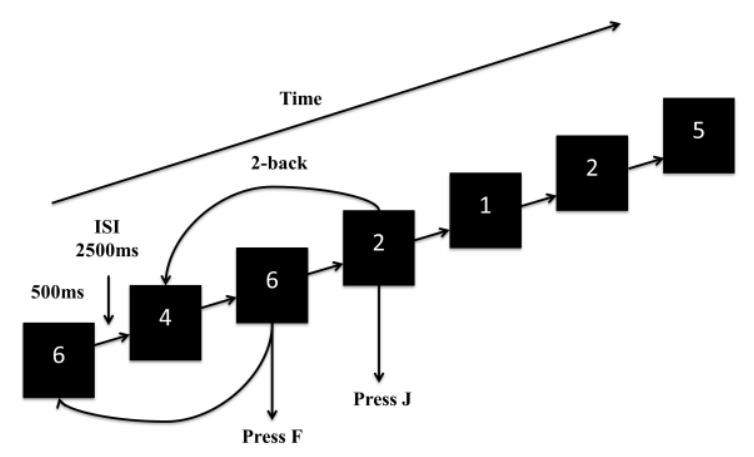

Figure.1 The 2-back test procedure

\section{Fatigue Questionnaire}

In order to assess the fatigue and attention states under the two conditions, participants were administered the fatigue questionnaire, which was based on the Japanese Association for Industrial Health (JAIH) study done in 1971 [29]. The questionnaire was a four-item self-report inventory designed to measure transient or fluctuating affecting states on four different scales as follows: mental clarity, attention concentration, sleepiness, and comprehensive assessment of fatigue (Fatigue sub-scale). Participants were required to describe their feelings using a scale ranging from 1 (very low) to 10 (extremely). In the fatigue questionnaire, a low score in mental clarity or attention concentration indicated a good state of mind or a good sustained attention state, respectively, accompanying a low fatigue level. A high score in sleepiness or comprehensive assessment of fatigue meant a high fatigue level.

\section{Electrophysiological Recording and Processing}

The EEG data was acquired using Brain Vision Recorder 1.03 software and QuikAmps 72 hardware (http://BrainProducts.com), recorded from $63 \mathrm{Ag} / \mathrm{AgCl}$ active electrode sensors mounted in an elastic cap (EasyCap, Brain Products $\mathrm{GmbH}$ ) with a standard 10/20 system layout. The electrooculogram (EOG) was recorded from two electrodes placed above and below the right eye and at the outer canthi. The ground electrode was positioned on the forehead at $\mathrm{AFz}$ and the reference electrode was positioned at FCz. Electrode impedances were kept below $5 \mathrm{k} \Omega$. Signals were recorded by using BrainVision Recorder (Brain ProductsGmbH) with an online low pass filter $(250 \mathrm{~Hz})$. When an electrode reached $70 \%$ saturation, a DC reset was applied. Sampling rate was $1000 \mathrm{~Hz}$ and signals were amplified in the range of $\pm 3.27 \mathrm{mV}$ at a resolution of $0.1 \mu \mathrm{V}$.

The EEG data were processed offline by using the BrainVision analyzer 2.0. software (Brain ProductsGmbH). TP9 and TP10 were selected as the new reference (Brain ProductsGmbH). Then EEG data were filtered by a band-pass filter from $0.01 \mathrm{~Hz}$ to $100 \mathrm{~Hz}$. After filtration, a semiautomatic inspection method was performed to inspect raw data. The gradient criteria were $50 \mu \mathrm{V} / \mathrm{ms}$. The maximal absolute difference allowed was $200 \mu \mathrm{V}$ and the interval length was $200 \mathrm{~ms}$. Amplitude was between -200 
$\mu \mathrm{V}$ and $200 \mu \mathrm{V}$. The lowest allowed activity was $0.5 \mu \mathrm{V}$. After raw data inspection, the EEG signals were corrected for eye-movement artifacts by using artifact rejection method based on the algorithm of Gratton and Coles [30]. The ocular correction was implemented in BrainVision analyzer 2.0 software. After filtering with a band-pass filter from $0.01 \mathrm{~Hz}$ to $35 \mathrm{~Hz}$, the data were segmented into: response-locked erroneous trials in the task. Artifact rejection removed trials where voltages exceeded $\pm 50 \mu \mathrm{V}$. Data were baseline-corrected from -400 to $-200 \mathrm{~ms}$ for response-locked epochs before computation of averages for each condition. The minimum number of trials used for ERN (0-120 ms) was not less than 5 [27], and the ERN mean amplitudes were quantified at $\mathrm{Fz} / \mathrm{Cz} / \mathrm{FC} 1 / \mathrm{FC} 2$ sites.

\section{Results}

\section{Fatigue Questionnaire Results}

Paired t-tests were used to compare the questionnaire data for the first 10 minutes and the last 10 minutes. Table 1 shows scores and statistical results for the fatigue questionnaire. The last 10 minutes' scores were substantially higher than the first 10 minutes' scores for 4 sub-scales. The mental clarity $(\mathrm{t}(32)=-2.153, \mathrm{p}=0.039)$ and sleepiness $(\mathrm{t}(32)=-2.379, \mathrm{p}=0.024)$ were higher for the last 10 minutes compared with the first 10 minutes. Furthermore, the attention score $(\mathrm{t}(32)=-2.150, \mathrm{p}=0.039)$ indicating the sustained attention level was significantly higher for the last 10 minutes, and the fatigue sub-scale score $(\mathrm{t}(32)=-2.673, \mathrm{p}=0.012)$ indicating fatigue level was also higher for the last 10 minutes.

Table 1 Mean summary data for fatigue questionnaire sub-scales

\begin{tabular}{|c|c|c|c|c|}
\hline & Mental clarity & Attention score & Sleepiness & Fatigue \\
\hline First $10 \mathrm{~min}$ & $2.7(2.0)$ & $3.1(2.1)$ & $3.4(2.2)$ & $3.4(2.0)$ \\
\hline Last $10 \mathrm{~min}$ & $4.4(2.4)^{*}$ & $4.6(2.0)^{*}$ & $5.1(2.0)^{*}$ & $5.3(2.2)^{*}$ \\
\hline
\end{tabular}

Note: $* \mathrm{p}<0.05 * * \mathrm{p}<0.01$. Standard deviations are provided in parentheses.

\section{Behavioral Results}

Paired t-tests were used to compare the behavioral data in the first 10 minutes and in the last 10 minutes. Table 2 shows the statistical results of behavioral data in the first 10 minutes and the last 10 minutes for the 2-back task. The error rate $(\mathrm{t}(16)=-3.432$, $\mathrm{p}=0.003$ ) in the last 10 minutes was significantly higher than in the first 10 minutes, and the accuracy rate $(\mathrm{t}(16)=2.537, \mathrm{p}=0.022)$ in the last 10 minutes was substantially lower than in the first 10 minutes. The RT correct $(\mathrm{t}(16)=3.641, \mathrm{p}=0.002)$ and the RT error $(\mathrm{t}(16)=2.793, \mathrm{p}=0.013)$ were remarkably smaller in the last 10 minutes than in the first 10 minutes; however, the omission rates $(\mathrm{t}(16)=-0.239, \mathrm{p}=0.814)$ were almost the same level in the first and last 10 minutes.

Table 2 Behavioral data in the first 10 minutes and the last 10 minutes of 100 minutes 2-back task

\begin{tabular}{|c|c|c|c|c|}
\hline & First 10min & Last 10min & $\mathrm{t}$ & Significance \\
\hline Error rate (\%) & $8.0(5.0)$ & $13.4(7.9)$ & -3.432 & 0.003 \\
\hline Accuracy rate (\%) & $90.6(5.3)$ & $82.3(12.7)$ & 2.537 & 0.022 \\
\hline Omission rate (\%) & $1.5(2.4)$ & $1.7(4.7)$ & -0.239 & 0.814 \\
\hline RT correct $(\mathrm{ms})$ & $670.3(134.3)$ & $543.4(130.2)$ & 3.641 & 0.002 \\
\hline RT error $(\mathrm{ms})$ & $810.2(209.7)$ & $629.2(273.9)$ & 2.793 & 0.013 \\
\hline
\end{tabular}

Note: Standard deviations are provided in parentheses. 


\section{ERP Results}

Figure 2 shows the grand average waveforms of ERN at $\mathrm{Fz} / \mathrm{Cz} / \mathrm{FC} 1 / \mathrm{FC} 2$ sites and topographic maps at $43 \mathrm{~ms}$ post response for the 2-back task in the first 10 minutes and the last 10 minutes with waveforms for erroneous responses (black lines indicate the first 10 minutes; red lines indicate the last 10 minutes). The ERN amplitudes were more negative in the first 10 minutes than in the last 10 minutes. Additionally, the ERN active areas were wider in the first 10 minutes than in the last 10 minutes for topographic maps.

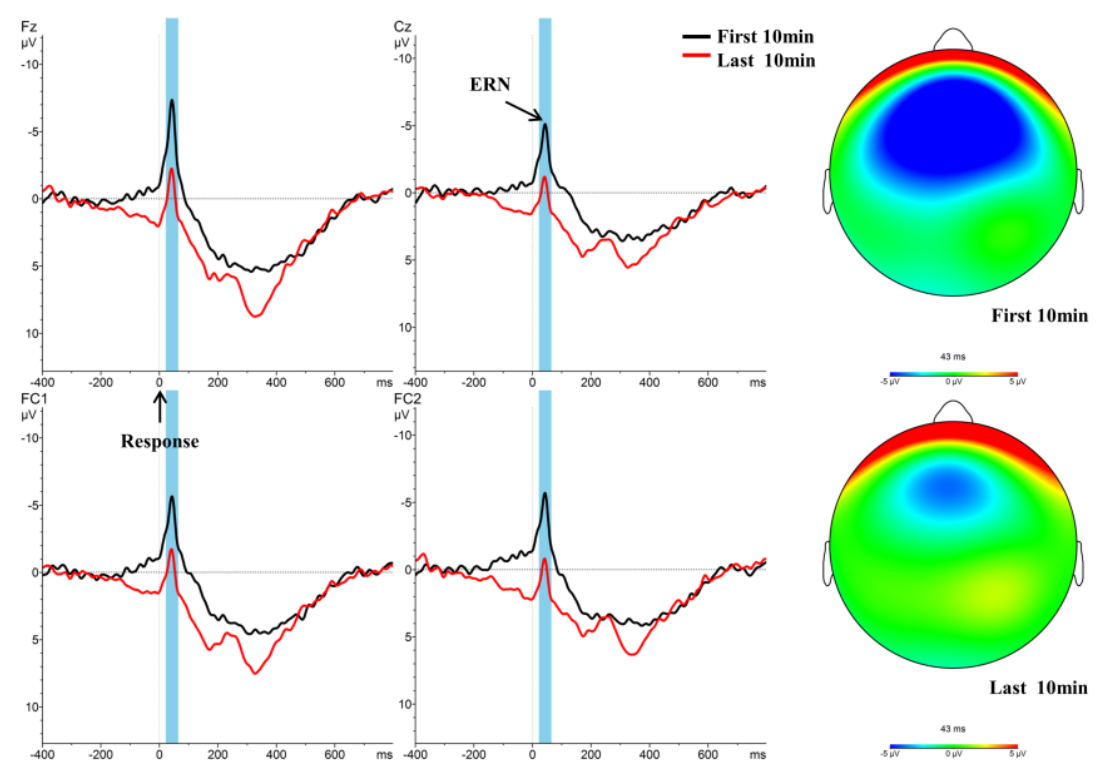

Figure.2 ERN waveforms and topographic maps of the 2-back task

As presented in Table 3, the ERN have been affected by mental fatigue. These differences have been analyzed by repeated measures ANOVAs with the between-subjects factor being Time-block (first 10 minutes or last 10 minutes) and within-subjects factors being Electrode (Fz, Cz, FC1, FC2). Across all conditions, the ERN mean amplitudes were substantially more negative in the first 10 minutes than in the last 10 minutes. There was a significant main effect of Time-block $(\mathrm{F}(1,32)=7.782$, $\left.\mathrm{p}=0.012, \eta_{\mathrm{p}}^{2}=0.291\right)$, but a main effect of Electrode $(\mathrm{F}(3,96)=1.345, \mathrm{p}=0.273$, $\left.\eta_{\mathrm{p}}{ }^{2}=0.066\right)$ was not found. There were no interactions of ElectrodexGroup ( $\mathrm{F}(3$, 96)<1.0).

Table 3 ERN mean amplitudes in the first 10 minutes and the last 10 minutes of 100 minutes 2-back task

\begin{tabular}{|c|c|c|}
\hline ERN mean amplitudes & First 10min & Last 10min \\
\hline $\mathrm{Fz}(\mu \mathrm{V})$ & $-2.21(3.30)$ & $1.15(5.36)$ \\
\hline $\mathrm{Cz}(\mu \mathrm{V})$ & $-1.91(3.02)$ & $1.16(3.19)$ \\
\hline $\mathrm{FC} 1(\mu \mathrm{V})$ & $-2.02(2.86)$ & $1.22(3.64)$ \\
\hline $\mathrm{FC} 2(\mu \mathrm{V})$ & $-1.86(2.97)$ & $1.81(5.09)$ \\
\hline
\end{tabular}

Note: Standard deviations are provided in parentheses.

\section{Correlation}

A bivariate Spearman correlation was found between the last 10 minutes ERN and the performance change trend in the 2-back task. The behavioral difference between the last 10 minutes and the first 10 minutes indicated the variation trend of the 2-back task 
performance. The ERN mean amplitude at the $\mathrm{Cz}$ site for the last 10 minutes was negatively correlated with $\Delta$ Accuracy rate $(\Delta$ Accuracy rate $=$ (Accuracy rate in the first $10 \mathrm{~min})$ - (Accuracy rate in the last $10 \mathrm{~min})$ ) in the 2-back task $(\mathrm{r}=0.772, \mathrm{p}<0.001)$. Based on the negative polarity of ERN, the correlation reflected that the participants whose accuracy rate decreased more had smaller ERN amplitudes on the 2-back task.

\section{Discussion}

The major findings could be summarized as follows: Across all conditions, the fatigue questionnaire revealed worse mental clarity and attention, greater sleepiness, and higher level of mental fatigue in the last 10 minutes, which confirmed a clear behavioral pattern. As hypothesized, smaller ERN amplitudes for the task demonstrated that the performance monitoring system was impaired during mental fatigue. There were significant correlations between the behavioral performance and the ERN amplitudes. This indicated that the participants who had invested more effort showed a stronger reduction in ERN amplitudes, which had further demonstrated that mental fatigue was induced successfully.

Error monitoring is a central function of cognitive control, including error detection and error evaluation, in order to monitor and regulate performance after erroneous responses. In line with previous studies, our research found support for the hypothesized attenuation in the ERN amplitudes as a result of mental fatigue for the 2-back task. The ERN can be considered an index of error monitoring. The results were accordance with previous studies [14,16-18,20,31]. These data reflect impairment in the performance monitoring system, which may more specifically reflect deficits in error detection and compensation processes [10], conflict detection processes [32], or the dopaminergic negative reinforcement learning system [33].

The correlation analysis can let us focus narrowly on objective behaviors and brain activations associated with the mechanism for mental fatigue on error monitoring. The accuracy rate and ERN amplitude were very highly correlated, suggesting that the lower the accuracy rate was, the smaller the ERN was. This relationship is in line with previous studies $[8,10]$, which reported that the ERNs were smaller when the participants made more errors and that those participants who had lower error rates produced larger ERNs. The relationship between the ERN and the accuracy rate could represent a "habituation" response to making errors. The ERN is reduced with a higher error rate, because errors become less salient. Since the ACC is a salience detector, error monitoring with ACC lesions due to mental fatigue would have reduced error awareness and diminished or absent ERNs following errors [34]. The habituation effect, which is reported by previous studies [8,33], could achieve the consequence of attenuation in the ACC activity because more and more repeated errors occurred and entered the ACC via the dopamine system.

\section{Conclusion}

This study has demonstrated the correlation of mental fatigue and error monitoring. Deficits in error monitoring were evidenced by the diminished ERN amplitudes and decreased performance level. This deficit was caused by mental fatigue. Participants clearly felt mental fatigue after the experiment, and their performances deteriorated with time on task. The close relationship between mental fatigue and error monitoring contributed to explaining the mechanism of the function between them. Understanding 
of the neural basis of this relationship is particularly important for preventing human error, selecting personnel, and training crews in spaceflight.

\section{Acknowledgement}

This study was financially supported by the Equipment Pre-research Foundation (51326050204).

\section{References}

[1] A.D. Swain, H.E. Guttmann. Handbook of human-reliability analysis with emphasis on nuclear power plant applications. Final report. No. NUREG/CR-1278; SAND-80-0200. Sandia National Labs., Albuquerque, NM (USA), 1983.

[2] E. Hollnagel. Cognitive reliability and error analysis method (CREAM): Elsevier; 1998.

[3] D. Shayler. Disasters and accidents in manned spaceflight: Springer Science \& Business Media; 2000.

[4] S.J. Johnstone, R.J. Barry, A.R. Clarke. Ten years on: a follow-up review of ERP research in attention-deficit/hyperactivity disorder. Clinical neurophysiology : official journal of the International Federation of Clinical Neurophysiology. 2013;124(4):644-57.

[5] A. Ramamoorthy, J.W. Brown. Performance Monitoring. In: Arthur W. Toga, editors. Brain Mapping. Waltham: Academic Press; 2015. pp. 355-359.

[6] M. Falkenstein, J. Hohnsbein, J. Hoormann, L. Blanke. Effects of crossmodal divided attention on late ERP components. II. Error processing in choice reaction tasks. Electroencephalography and clinical neurophysiology. 1991;78(6):447-55.

[7] M. Falkenstein, J. Hoormann, S. Christ, J. Hohnsbein. ERP components on reaction errors and their functional significance: a tutorial. Biological psychology. 2000;51(2):87-107.

[8] G. Hajcak, J.S. Moser, N. Yeung, R.F. Simons. On the ERN and the significance of errors. Psychophysiology. 2005;42(2):151-60.

[9] R. Grützmann, T. Endrass, J. Klawohn, N. Kathmann. Response accuracy rating modulates ERN and Pe amplitudes. Biological psychology. 2014;96:1-7.

[10] W.J. Gehring, B. Goss, M.G. Coles, D.E. Meyer, E. Donchin. A neural system for error detection and compensation. Psychological science. 1993;4(6):385-90.

[11] A. Riesel, A. Weinberg, T. Endrass, A. Meyer, G. Hajcak. The ERN is the ERN is the ERN? Convergent validity of error-related brain activity across different tasks. Biological psychology. 2013;93(3):377-85.

[12] J. Wu, Y. Yuan, H. Duan, S. Qin, T.W. Buchanan, K. Zhang, et al. Long-term academic stress increases the late component of error processing: An ERP study. Biological psychology. 2014; 99:77-82.

[13] V. van Veen, C.S. Carter. The timing of action-monitoring processes in the anterior cingulate cortex. Journal of cognitive neuroscience. 2002; 14(4):593-602. 
[14]N. Yeung, M.M. Botvinick, J.D. Cohen. The neural basis of error detection: conflict monitoring and the error-related negativity. Psychological review. 2004; 111(4):931.

[15] D. Wiswede, T.F. Münte, J. Rüsseler. Negative affect induced by derogatory verbal feedback modulates the neural signature of error detection. Social Cognitive and Affective Neuroscience. 2009:nsp015.

[16]G. Hughes, N. Yeung. Dissociable correlates of response conflict and error awareness in error-related brain activity. Neuropsychologia. 2011;49(3):405-15.

[17]M.E. Maier, N. Yeung, M. Steinhauser. Error-related brain activity and adjustments of selective attention following errors. NeuroImage. 2011;56(4):2339-47.

[18] M.M. Lorist, M.A. Boksem, K.R. Ridderinkhof. Impaired cognitive control and reduced cingulate activity during mental fatigue. Brain research Cognitive brain research. 2005;24(2):199-205.

[19] M.A. Boksem, T.F. Meijman, M.M. Lorist. Effects of mental fatigue on attention: an ERP study. Brain research Cognitive brain research. 2005;25(1):107-16.

[20] M.A. Boksem, T.F. Meijman, M.M. Lorist. Mental fatigue, motivation and action monitoring. Biological psychology. 2006;72(2):123-32.

[21]P.E. Clayson, A. Clawson, M.J. Larson. The effects of induced state negative affect on performance monitoring processes. Social cognitive and affective neuroscience. 2012;7(6):677-88.

[22] S. Johannes, B.M. Wieringa, W. Nager, D. Rada, R. Dengler, H.M. Emrich, et al. Discrepant target detection and action monitoring in obsessive-compulsive disorder. Psychiatry Research: Neuroimaging. 2001;108(2):101-10.

[23] A.T. Bates, K.A. Kiehl, K.R. Laurens, P.F. Liddle. Error-related negativity and correct response negativity in schizophrenia. Clinical Neurophysiology. 2002;113(9):1454-63.

[24] G. Hajcak, R.F. Simons. Error-related brain activity in obsessive-compulsive undergraduates. Psychiatry research. 2002;110(1):63-72.

[25] J. Wiersema, J. van der Meere, H. Roeyers. ERP correlates of impaired error monitoring in children with ADHD. Journal of neural transmission. 2005;112(10):1417-30.

[26] R.G. O'Connell, P.M. Dockree, M.A. Bellgrove, A. Turin, S. Ward, J.J. Foxe, et al. Two types of action error: electrophysiological evidence for separable inhibitory and sustained attention neural mechanisms producing error on go/no-go tasks. Journal of Cognitive Neuroscience. 2009;21(1):93-104.

[27] D.M. Pfabigan, N.M. Pintzinger, D.R. Siedek, C. Lamm, B. Derntl, U. Sailer. Feelings of helplessness increase ERN amplitudes in healthy individuals. Neuropsychologia. 2013;51(4):613-21.

[28] M. Tanaka, Y. Shigihara, A. Ishii, M. Funakura, E. Kanai, Y. Watanabe. Effect of mental fatigue on the central nervous system: an electroencephalography study. Behavioral and brain functions. 2012;8(1):48-49. 
[29] H. Yoshitake. Methodological study on the inquiry into subjective symptoms of fatigue. J Sci Labour. 1971;47:797-802.

[30] G. Gratton, M.G. Coles, E. Donchin. A new method for off-line removal of ocular artifact. Electroencephalography and clinical neurophysiology. 1983;55(4):468-84.

[31] Y. Xiao, F. Ma, Y. Lv, G. Cai, P. Teng, F. Xu, et al. Sustained attention is associated with error processing impairment: evidence from mental fatigue study in four-choice reaction time task. PloS one. 2015;10(3):e0117837.

[32] M. Botvinick, L.E. Nystrom, K. Fissell, C.S. Carter, J.D. Cohen. Conflict monitoring versus selection-for-action in anterior cingulate cortex. Nature. 1999;402(6758):179-81.

[33] C.B. Holroyd, M.G. Coles. The neural basis of human error processing: reinforcement learning, dopamine, and the error-related negativity. Psychological review. 2002;109(4):679-709.

[34]D.H. Mathalon, S.L. Whitfield, J.M. Ford. Anatomy of an error: ERP and fMRI. Biological psychology. 2003;64(1-2):119-41. 